\title{
A thermal parametric study of non-evaporative spray cooling process
}

\author{
Abdessalam Otmani ${ }^{1}$,Hocine $\mathrm{Mzad}^{2, *}$, and Kamel Bey ${ }^{2}$ \\ ${ }^{1}$ Laboratory of Industrial Mechanics, Badji Mokhtar University of Annaba, DZ-23000, Algeria \\ ${ }^{2}$ Department of Mechanical Engineering, Badji Mokhtar University of Annaba, P.O. Box 12, DZ-23000, Algeria
}

\begin{abstract}
Ordinary water spray cooling is connected with very high temperatures where heat transfer during evaporation plays a key role. However, during cooling without phase change, the behaviour of the spray cooling parameters is rarely considered. The purpose of this paper is to study the influence of spray hydrodynamic parameters on heat transfer without liquid phase change during the cooling of an aluminium 3003-H18 plate at a temperature of $92{ }^{\circ} \mathrm{C}$. First of all, the flow rate was varied from 0.497 up to $1 \mathrm{l} / \mathrm{min}$. Then, the inlet pressure varied from 0.7 to 2.1 bars. The influence of nozzle-to-target distance is also tested since the simulations were carried out in a wide height range, from $100 \mathrm{~mm}$ to $505 \mathrm{~mm}$. The present simulation was achieved using the version 5.2 of COMSOL Multiphysics code.
\end{abstract}

\section{Introduction}

Optimizing the hydrodynamic parameters of the jet during nozzle design helps to obtain better dispersion and economy in water consumption. The nozzle regulates the flow rate, atomizes the liquid into droplets, and disperses the droplets in a specific pattern. The proper tip nozzle must be selected for the desired job; no single nozzle can meet all the spraying needs.

The thermal performance during spray injection of liquid onto surfaces is normally represented by the associated heat transfer coefficient or the Nusselt number, which is a measure of the cooling rate due to forced convection. Characterization of the heat transfer coefficient is important for determining the required cooling capacity and corresponding internal properties of the product. For this reason, a vast amount of work has been devoted to parameterize or model the heat transfer coefficient during spray cooling in different flow regimes $[1,2]$.

A correlation was established for the quantification of water jet dispersion on the totality of a plane surface in the function of the spray nozzle dimensions and hydrodynamic parameters. Subsequently, numerical simulations based on the above experimental results were conducted to investigate the effect of the waterspray characteristics on the heat transfer mechanisms involved during cooling of steel plates at high temperatures $[3,4]$.
A model to predict the heat and mass transfer in spray cooling was presented with consideration of droplet-film impaction, film formation, film motion, bubble boiling, droplet-bubble interaction, bulk air convection and radiation [5].

If multiple nozzles are used in a conveyorized application, the overlapping liquid distribution pattern of the nozzles needs to be considered because the process may depend strongly on the relative local volume flux of the spray. In order to understand the fluid behaviour in the overlap region, a hydrodynamic study was proposed using a FORTRAN programming based on experimental correlations of pulverized water jets [6].

The effects of droplet velocity non-uniformity, Sauter mean diameter distribution non-uniformity, droplet number non-uniformity, and heating power on the fluid film thickness, fluid film velocity, and surface temperature distribution were investigated, and then the surface temperature non-uniformity correlations in nonboiling regime and nucleate boiling regime were correlated [7].

The heat transfer characteristics of air water spray impingement cooling of stationary steel plate were experimentally investigated. The controlling parameters taken during the experiments were fluid pressure, water flow rate, nozzle tip to target distance and mass impingement density. The effects of the controlling parameters on the cooling rates were critically examined during spray impingement cooling [8].

\footnotetext{
* Corresponding author: h_mzad@yahoo.fr
} 
A computational fluid dynamics (CFD) assessed the potential and performance of evaporative cooling by water spray system with a hollow-cone nozzle configuration [9].

Nizetic et al. [10] proposed an alternative cooling technique for photovoltaic (PV) panels that includes a water spray application over panel surfaces. Both sides of the PV panel were cooled simultaneously, to investigate the total water spray cooling effect on the PV panel performance in circumstances of peak solar irradiation levels. Furthermore, it was also possible to decrease panel temperature from an average $54{ }^{\circ} \mathrm{C}$ (noncooled PV panel) to $24{ }^{\circ} \mathrm{C}$ in the case of simultaneous front and backside PV panel cooling.

The purpose of this study is to highlight, by simulation under COMSOL Multiphysics, the influence of hydrodynamic parameters, namely the nozzle height, water flow rate and spraying pressure in order to evaluate the impact of nozzle characteristics and operating conditions on cooling performance of the spray system.

\section{Modelling and Simulation}

In the simplest case, a single nozzle is employed to distribute liquid in a defined pattern to cover a surface or fill a spatial volume in a prescribed manner. Hydraulic atomizers depend on the internal geometry and liquid pressure alone to produce a desired pattern. The exact definition of the spray characteristics and the specification of the operation parameters are the first two steps to be taken when a spray nozzle is being designed. Using the commercial software COMSOL Multiphysics 5.2 we achieved a specific application, coupled between heat transfer and fluid flow interface, for the simulation of a cooling process beneath liquid sprayer. The objective is to cool an aluminium $3003-\mathrm{H} 18$ plate (Tab. 1) with $6 \mathrm{~mm}$ thickness and an area of $1200 \mathrm{~mm}^{2}$, initially at the temperature of $92{ }^{\circ} \mathrm{C}$.

A single nozzle fixed at the centre upon the plate, delivers the simulated water spray in an ambience at atmospheric pressure and a temperature of $20{ }^{\circ} \mathrm{C}$. The nozzle geometry and meshing are shown on figure 1 .The modelling section is executed in two steps, the fluid flow model and heat transfer model.
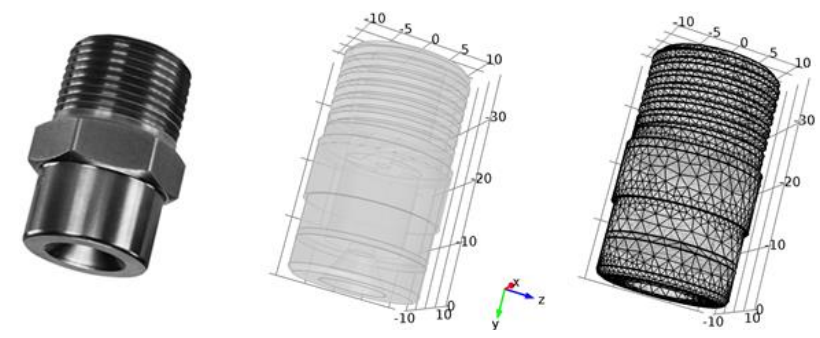

Table 1. Aluminium 3003-H18 Properties.

\begin{tabular}{|l|l|}
\hline \multicolumn{1}{|c|}{ Parameter } & \multicolumn{1}{c|}{ Value } \\
\hline Specific heat capacity, $C_{p}$ & $890 \mathrm{~J} /(\mathrm{kg} . \mathrm{K})$ \\
\hline Density, $\rho$ & $2.8 \mathrm{~g} / \mathrm{cm}^{3}$ \\
\hline Thermal conductivity, $k$ & $180 \mathrm{~W} /(\mathrm{m} . \mathrm{K})$ \\
\hline Thermal expansion coeff., $\alpha$ & $2.3 \mathrm{E}-51 / \mathrm{K}$ \\
\hline Melting point, $T_{m}$ & $640^{\circ} \mathrm{C}$ \\
\hline
\end{tabular}

The resolution of the Navier-Stokes equations was achieved for laminar fluid flow and stationary regime. The simulation was realized in two steps. The first one is the simulation of the laminar flow around the aluminium plate, inside and outside the nozzle. The obtained results were used in the second step, which is the simulation of the laminar bubbly flow (Fig. 2). Using a two-phase Euler-Euler model, a momentum balance equation and a continuity equation describe the dynamics of each phase.

Therefore, a combination of the momentum and the continuity equations gives:

$$
\frac{\partial \rho_{g} \phi_{g}}{\partial t}+\nabla \cdot\left(\phi_{g} \rho_{g}\left(u_{l}+u_{s l i p}\right)\right)=\nabla \cdot\left(\frac{\tilde{\mu} \rho_{g}}{\rho_{l}} \nabla \phi_{g}\right)-m_{g l}
$$

The gas phase transport equation is expressed as:

$$
\frac{\partial \rho_{g} \phi_{g}}{\partial t}+\nabla \cdot\left(\phi_{g} \rho_{g} u_{g}\right)=-m_{g l}
$$

The gas velocity $u_{g}$ is the sum of the following velocities:

$$
u_{g}=u_{l}+u_{\text {slip }}+u_{d r i f t}
$$

The liquid volume fraction is calculated from:

$$
\phi_{l}=1-\phi_{g}
$$

The drift velocity expression is:

$$
u_{d r i f t}=-\frac{\tilde{\mu}}{\rho_{l}} \frac{\nabla \phi_{g}}{\phi_{g}}
$$

Where $\tilde{\mu}$ is an effective viscosity causing the drift.

A diffusive term is introduced in the gas transport equation (Eq. 2), which is implemented in the fluid-flow model.

Fig. 1. BETE WL6 nozzle. 


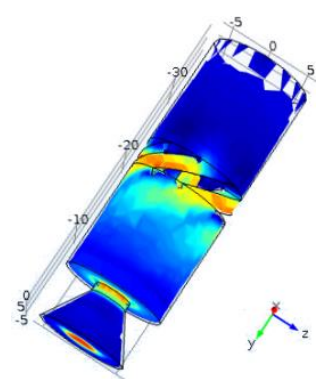

Surface: Velocity magnitude $(\mathrm{m} / \mathrm{s})$
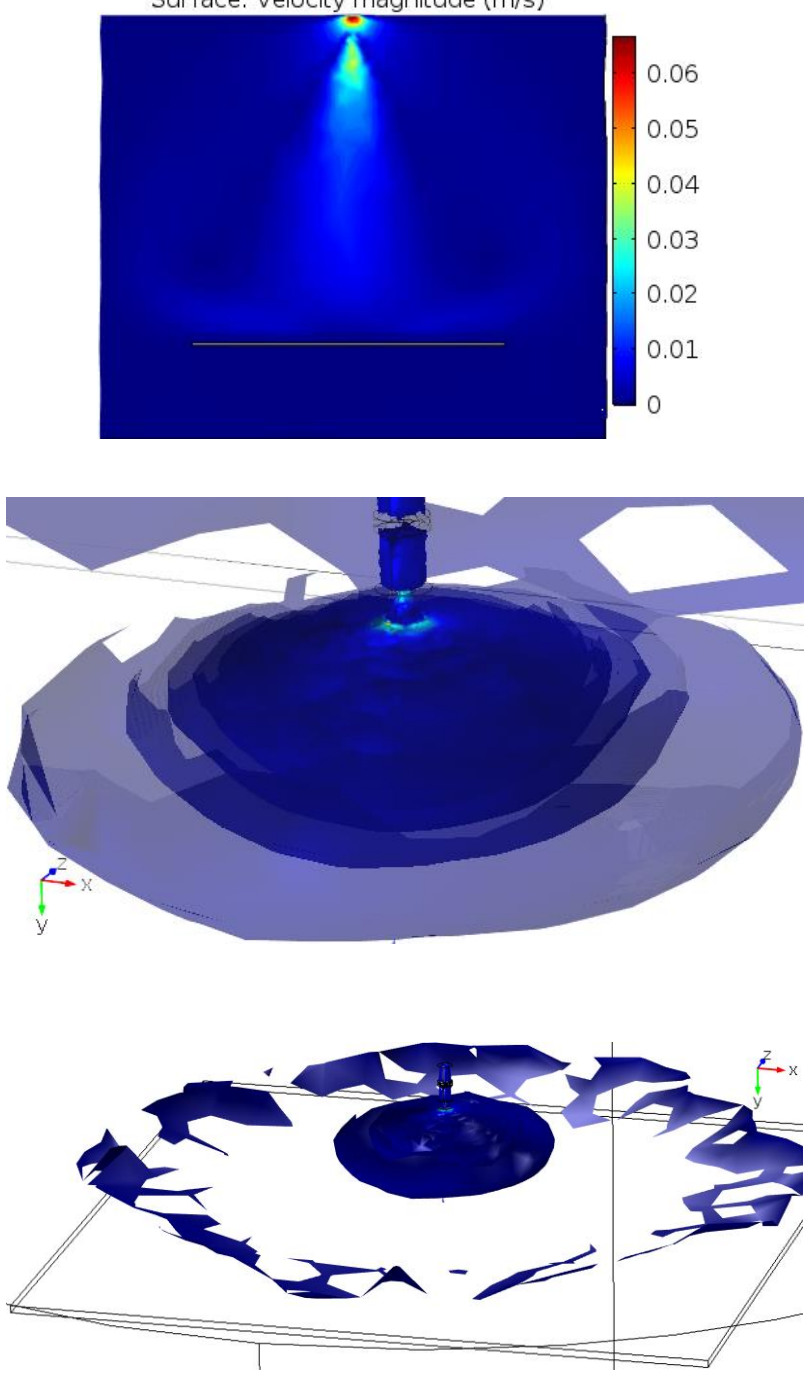

Fig. 2. Simulation of water spray dispersion.

The temperature distribution in a three-dimensional space within the considered solid is described by the equation of the heat conduction:

$$
\rho C_{p} \frac{\partial T}{\partial t}+\rho C_{p} u \nabla T+\nabla q=0
$$

By solving the heat equation, we evaluate the maximum plate temperature and the average convection heat flux variation.
There is no liquid evaporation, because the maximum plate temperature is below the saturation temperature of water.

The initial water supply temperature was $20^{\circ} \mathrm{C}$. The heat transfer in fluid flow is solved using the equation:

$$
\rho C_{p}\left(\frac{\partial T}{\partial t}+u . \nabla T\right)+\nabla .\left(q+q_{r}\right)=Q_{p}+Q_{V d}
$$

Equation in which:

$$
\begin{gathered}
Q_{p}=\alpha_{p} T\left(\frac{\partial p}{\partial t}+u . \nabla p\right) \\
\alpha_{p}=-\frac{1}{\rho} \frac{\partial \rho}{\partial T}
\end{gathered}
$$

and,

$$
Q_{V d}=\tau: \nabla u
$$

The above term represents viscous heat dissipation in the fluid. For the gas density estimation, the model use the ideal gas law, i.e. the thermal expansion coefficient $\alpha_{p}=1 / T$.

The grid generation was executed resulting in a free tetrahedral meshing which consists of 41640 domain elements. A finer grid is applied for the nozzle and extracoarse grid for the aluminium plate and the controlled volume of fluid. The chosen computational time stepping is $\Delta t=1 \mathrm{~min}$.

\section{Discussion of the results}

The purpose of this simulation is to highlight the influence of three important hydrodynamic parameters, involved in water spraying cooling process, on heat transfer behaviour of an aluminium plate at a temperature of $92^{\circ} \mathrm{C}$.

Figure 3 illustrate the effect of water mass-flow rate on the temperature evolution, the tested flow values are 0.497, 0.7 and 1 litre/min. The results show a rapid decrease of the plate temperature during the first five minutes. The rest of time there is a very slow decrease of the temperature until $t=60 \mathrm{~min}$, this is due to the insignificant temperature difference between the plate and the impinging water. To disclose the influence of flow variation on heat transfer, an enlargement (Fig. 3) makes it possible to distinguish the flow rate increase effect on the cooling stretch.

Convection heat dissipation is also influenced by water-spray flow, since we notice a decrease in convective heat flux which means the internal energy fall and consequently the diminution of wall temperature (Fig. 4).

From the graphical results obtained on figure 5, it appears that the optimum cooling pressure is 2.1 bars among those applied namely 0.7 bars, 1.9 bars and 2.1 bars. In the current context, it is the pressure that offers the best heat transfer resulting in a drop of the plate temperature, at $\mathrm{t}=5 \mathrm{~min}$ the temperature value recorded is $53{ }^{\circ} \mathrm{C}$. The decrease in temperature is explained by the 
heat flux released by forced convection with an average value of approximately $900 \mathrm{~W} / \mathrm{m}^{2}$ during the entire cooling period (Fig. 6). For example, at $P=0.7$ bars corresponds a heat release of $17 \mathrm{~W} / \mathrm{m}^{2}$, which represents a derisory value compared to the $1100 \mathrm{~W} / \mathrm{m}^{2}$ evacuated at the optimal pressure $P=2.1$ bars.

Figures 7 and 8 aims to investigate the influence of the nozzle-to-target distance on the cooling spray performance, to be done, we considered four different positions: 100, 191, 330 and $505 \mathrm{~mm}$. The role of this parameter appears clearly on the graph since we note the significant influence of the nozzle position on the plate temperature evolution (Fig. 7). In this study, the value $H$ $=505 \mathrm{~mm}$ is the optimum height and it is the one that ensures the fastest cooling. The effect of the height also appears on the convection heat flux (Fig. 8), indeed for $H=505 \mathrm{~mm}$ the significant surface covered by the spray enhances the convection heat transfer. Obviously, the minimum heat flux is recorded for $H=100 \mathrm{~mm}$.

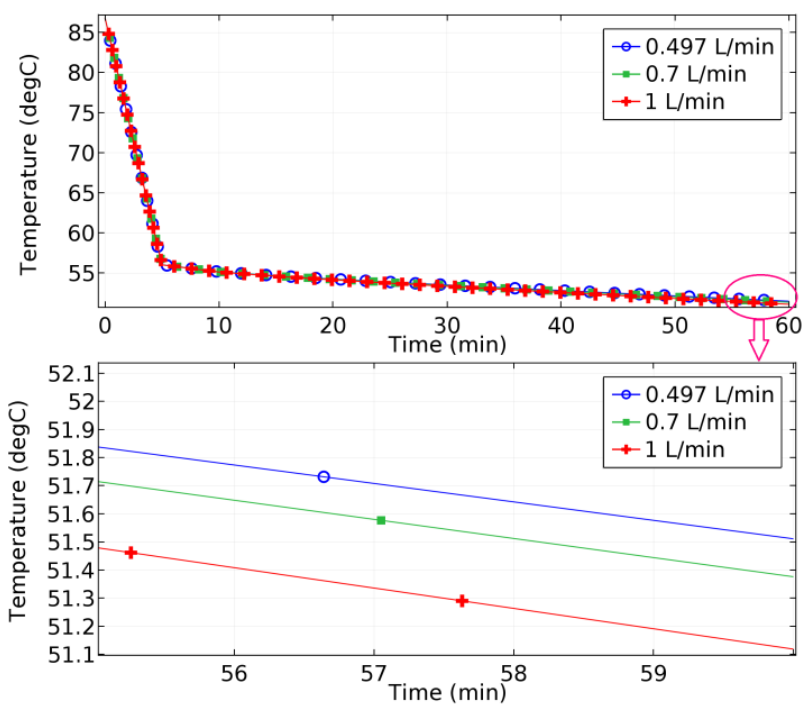

Fig. 3. Plate temperature decrease profile under flow rate variation.

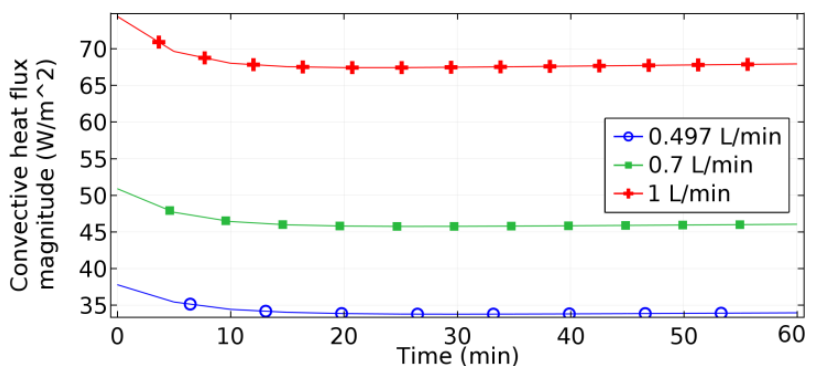

Fig. 4. Flow rate effect on temporary heat flux.

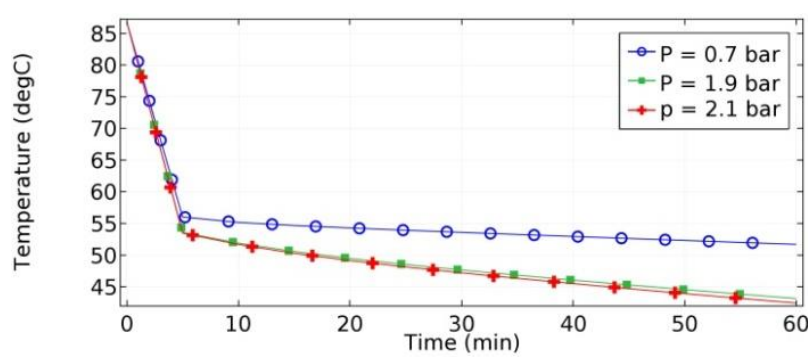

Fig. 5. Evolution of plate temperature under spray pressure influence.

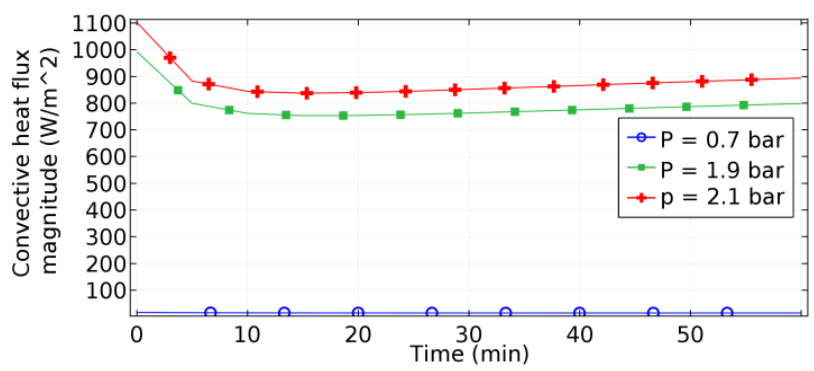

Fig. 6. Influence of spraying pressure on convection heat transfer.

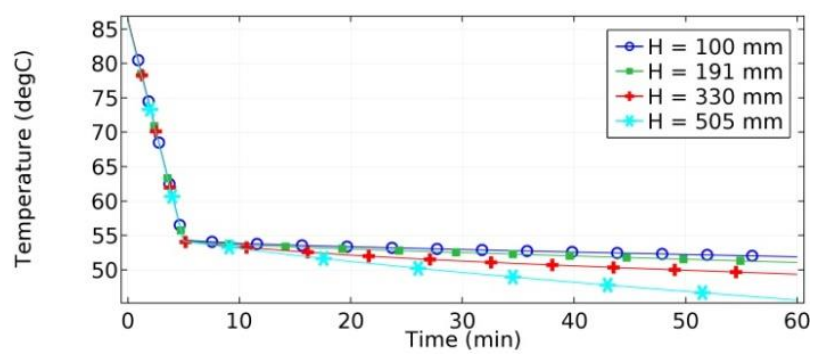

Fig. 7. Effect of nozzle height on plate temperature.

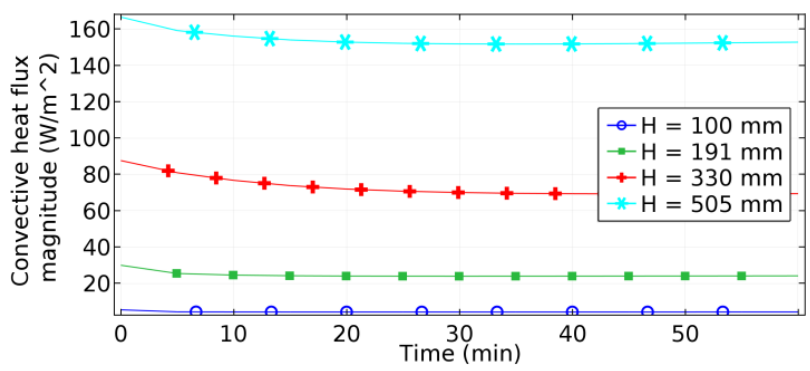

Fig. 8. Convection heat flux evolution with nozzle height. 


\section{Sum-up and conclusion}

This paper deals with influence of spray cooling process parameters on the thermodynamic behaviour of fluid flow and an aluminium plate at the temperature of $92{ }^{\circ} \mathrm{C}$. Unlike previous studies devoted solely to intensive cooling of highly heated surfaces, the present study makes it possible to observe the evolution of thermal parameters during laminar water jet impingement cooling of surface at a temperature below the saturation temperature of the cooling fluid.

The results of the simulation discussed in this paper signal the need to provide more insight for parametric analysis during spray cooling process involving the applications in such case of surface temperature, below $100{ }^{\circ} \mathrm{C}$.

Increase in electrical efficiency depends primarily on cooling techniques. It is well known that a decrease in the PV panel temperature will lead to an increase in electrical efficiency. Besides, high temperatures reduce the time-life of the photovoltaic system. In order to increase the average efficiency in conventional PV panels it is necessary to have more efficient backside thermal dissipation. The backside surface is usually of aluminium made and the analysis presented in this study considering aluminium 3003-H18 cooling allows instantaneously the control and monitoring of photovoltaic panels temperature.

\section{References}

1. B.W. Webb, C.F. Ma, Advances in Heat Transfer, 26, 105-217 (1995)

2. J. Kim, Int. J. of Heat and Fluid Flow, 28, 753-767 (2007)

3. M. Tebbal, H. Mzad, Forsch. im Ingenieurwesen, 68, 126-132 (2004)

4. H. Mzad, M. Tebbal, Heat Mass Transfer, 45, 287295 (2009)

5. R. Zhao, W.L. Cheng, Q.N. Liu, H.L. Fan, Heat and Mass Transfer, 46, 821-829 (2010)

6. H. Mzad, M. Elguerri, Atomization and Sprays, 22, 447-460 (2012)

7. W.L. Cheng, F.Y. Han, Q.N. Liu, R. Zhao, Int. J. Heat Mass Transfer, 55, 5357-5366 (2012)

8. H. Mzad, R. Khelif, Procedia Engineering, 157, 106-113 (2016)

9. H. Montazeri, B. Blocken, J.L.M. Hensen, Building and Environment, 83, 129-141 (2015)

10. S. Nizetic, F. Grubisic-Cabo, I. Marinic-Kragic, A.M. Papadopoulos, Energy, 111, 211-225 (2016) 